\title{
Performance of two-dimensional Doppler echocardiography for the assessment of infarct size and left ventricular function in rats
}

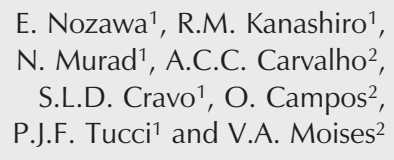

E. Nozawaํ', R.M. Kanashiro ${ }^{1}$, N. Murad ${ }^{1}$, A.C.C. Carvalho ${ }^{2}$, S.L.D. Cravo ${ }^{1}$, O. Campos ${ }^{2}$, P.J.F. Tucci ${ }^{1}$ and V.A. Moises $^{2}$

\author{
${ }^{1}$ Disciplina de Fisiologia Cardiovascular, ${ }^{2}$ Disciplina de Cardiologia ${ }^{2}$, \\ Escola Paulista de Medicina, Universidade Federal de São Paulo, \\ São Paulo, SP, Brasil
}

\section{Correspondence \\ V.A. Moises \\ Disciplina de Cardiologia \\ EPM, UNIFESP \\ Rua Botucatu, 740, 20 andar \\ 04023-900 São Paulo, SP \\ Brasil \\ Fax: +55-11-5571-0047 \\ E-mail: vmoises@cardiol.br}

Research supported by CNPq (No. 300.692/80-3NV) and FAPESP (No. 99/0045-3).

Received March 23, 2005

Accepted January 2, 2006

\begin{abstract}
Although echocardiography has been used in rats, few studies have determined its efficacy for estimating myocardial infarct size. Our objective was to estimate the myocardial infarct size, and to evaluate anatomic and functional variables of the left ventricle. Myocardial infarction was produced in 43 female Wistar rats by ligature of the left coronary artery. Echocardiography was performed 5 weeks later to measure left ventricular diameter and transverse area (mean of 3 transverse planes), infarct size (percentage of the arc with infarct on 3 transverse planes), systolic function by the change in fractional area, and diastolic function by mitral inflow parameters. The histologic measurement of myocardial infarction size was similar to the echocardiographic method. Myocardial infarct size ranged from 4.8 to $66.6 \%$ when determined by histology and from 5 to $69.8 \%$ when determined by echocardiography, with good correlation $(\mathrm{r}=0.88$; $\mathrm{P}<0.05$; Pearson correlation coefficient). Left ventricular diameter and mean diastolic transverse area correlated with myocardial infarct size by histology $(\mathrm{r}=0.57$ and $\mathrm{r}=0.78 ; \mathrm{P}<0.0005)$. The fractional area change ranged from $28.5 \pm 5.6$ (large-size myocardial infarction) to $53.1 \pm 1.5 \%$ (control) and correlated with myocardial infarct size by echocardiography $(\mathrm{r}=-0.87 ; \mathrm{P}<0.00001)$ and histology $(\mathrm{r}=-0.78 ; \mathrm{P}$ $<00001)$. The E/A wave ratio of mitral inflow velocity for animals with large-size myocardial infarction $(5.6 \pm 2.7)$ was significantly higher than for all others (control: $1.9 \pm 0.1$; small-size myocardial infarction: $1.9 \pm 0.4$; moderate-size myocardial infarction: $2.8 \pm 2.3$ ). There was good agreement between echocardiographic and histologic estimates of myocardial infarct size in rats.
\end{abstract}

\section{Introduction}

The rat model of myocardial infarction has been used for decades in many research laboratories to study left ventricular function (1-3),
Key words

- Rats

- Myocardial infarction size

- Systolic and diastolic

function

- Doppler echocardiography 
acquired or congenital diseases, particularly in patients with myocardial infarction during the acute phase and for late follow-up (11). The development of high definition echocardiographic imaging systems with high frequency transducers has permitted the study of the heart in small animal models of myocardial infarction in which Doppler echocardiography has been used to evaluate systolic and diastolic function, infarct size (12-14) and left ventricular remodeling (15). The methods used to obtain such information varied among studies, particularly for infarct size. Some studies have dealt with the estimation of myocardial infarct size in rats by echocardiography, and usually have not directly compared the measurement obtained by echocardiography with that obtained by histology. On this basis, the present study was undertaken to evaluate the ability of two-dimensional Doppler echocardiography to estimate the infarct size determined by histology in rats and its consequences for other anatomic and functional variables.

\section{Material and Methods}

The animals were cared for in compliance with the "Principles of Laboratory Animal Care" formulated by the National Institutes of Health (publication No. 96-23, revised, 1996). The study was approved by the local committee for animal research.

Myocardial infarction production. The surgical procedure to produce myocardial infarction was performed in 72 female Wistar rats weighing 156 to $241 \mathrm{~g}$ according to a well-accepted technique $(1,2)$. Briefly, after anesthesia with intraperitoneal (ip) ketamine $(100 \mathrm{mg} / \mathrm{kg})$ plus xylazine $(10 \mathrm{mg} / \mathrm{kg})$ a left thoracotomy was performed. The heart was exteriorized and the left anterior descending coronary artery ligated with 6-0 polypropylene suture. The beating heart was then quickly returned to its original position and the thorax immediately closed. Of the 72 animals submitted to this protocol, 29 died during the period of observation (5 weeks), and 43 remained in the study.

Echocardiography. The procedure was performed 5 weeks after surgery for myocardial infarction production using an Apogee Cx Ultrasound System (ATL Inc., Ambler, PA, USA) with a 7.5-MHz transducer at 3 $\mathrm{cm}$ depth and fundamental imaging. For image acquisition, the animals were anesthetized with intraperitoneal xylazine $(10 \mathrm{mg} /$ $\mathrm{kg})$ plus ketamine $(100 \mathrm{mg} / \mathrm{kg})$. Two-dimensional and $\mathrm{M}$-mode images from the parasternal long and short axis, and apical views were obtained and recorded on videotape for later review. Transverse images were obtained at three levels: basal (at the tip of the mitral valve leaflets), middle (at the papillary muscle level), and apical (distal to papillary muscle but before the cap of the cavity). Myocardial infarction was defined by echocardiography as any segmental wall motion abnormality such as hypokinesis, akinesis and dyskinesis.

Imaging analysis and measurements. These procedures were performed by one observer (V.A.M.) blind to the animal's condition and to the histological measurements. The videotapes were reviewed and the best images were digitized into the Image Vue DCR 1.60 System (Nova MicroSonics, Mahwah, NJ, USA) for off-line measurements.

Left ventricular dimensions. The enddiastolic diameter of the left ventricle (mm) was measured from the parasternal long or short axis views using M-mode or two-dimensional images $(16,17)$. The end-diastolic and systolic transverse areas $\left(\mathrm{cm}^{2}\right)$ of the left ventricle were obtained by tracing the endocardial border of basal, middle and apical short axis images. The value for each plane was the mean of three measurements and the final value of the end-diastolic and systolic areas for each animal was the mean of all three planes.

Myocardial infarct size. On each echocardiographic transverse plane (basal, middle and apical) the arc corresponding to the segments with infarction (AI) and to the total 
perimeter of the endocardial border (PE) were measured three times, at end-diastole, and the infarct size (IS) was estimated as IS $(\%)=\mathrm{AI} / \mathrm{PE} \times 100$. The final value of myocardial infarction size for each animal is reported as the mean IS obtained from the three planes.

Left ventricular systolic function. This parameter was estimated using the fractional area change (FAC), which was calculated as the difference between the diastolic area and systolic area divided by the diastolic area, multiplied by 100 and reported as percentage (\%) (26\%) (17). The end-diastolic and systolic areas were measured as described above for left ventricular dimensions.

Left ventricular diastolic function. This parameter was analyzed using the values derived from mitral diastolic inflow and left ventricular outflow tract velocity curves by pulsed Doppler (11). From the four-chamber view the sample volume was positioned at the tip of the mitral valve to obtain the mitral diastolic flow velocity, which were used to measure the peak $\mathrm{E}$ and $\mathrm{A}$ wave velocities $(\mathrm{cm} / \mathrm{s})$, the ratio between them $(\mathrm{E} /$ A ratio) and the deceleration time (ms). With the sample volume positioned between the mitral inflow and left ventricular outflow tract, a velocity profile was obtained that allowed to measure the isovolumic relaxation time as the interval (ms) between the end of left ventricular outflow tract velocity and the beginning of mitral inflow velocity.

Histological determination of myocardial infarct size. After the echocardiographic study the animals were anesthetized with urethane $(40 \mathrm{mg} / \mathrm{kg}$, ip $)$, the thorax was opened and the heart excised. The atria, remnants of the great vessels and the free wall of the right ventricle were extirpated. The left ventricle was placed in $10 \%$ formalin and, after fixation, five transverse slices of similar thickness were obtained, dehydrated with alcohol, embedded in paraffin and stained with hematoxylin-eosin and Masson trichrome. The three sections corre- sponding to the basal, middle and apical planes of the left ventricle were used for histological determination of myocardial infarct size as described $(1,2)$. The images of the histological sections were obtained with a camera connected to a microscope and then digitized. Four measurements were made on each transverse section: a) the epicardial and endocardial border circumferences of the left ventricle and $b$ ) the length of the epicardial and endocardial arcs of the region with myocardial infarction. The value for each section was the mean of three sets of measurements of the endocardial and epicardial borders and the infarct size for each animal was estimated as the mean percentage of the length of the arc with infarct in relation to the circumference of the left ventricle on the three transverse planes, similar to the echocardiographic method described. This histological measurement was used to classify the animals as having a small (4 to $\leq 29.9 \%$ ), medium ( $>30$ and $\leq 39.9 \%$ ) or large $(\geq 40 \%)$ myocardial infarction. Animals with a histological infarct size $<4 \%$ or without signs of myocardial infarction were included in the control group.

\section{Statistical analysis}

Data are reported as means $\pm \mathrm{SD}$ and range. The infarct sizes determined by echocardiography and histology were compared by simple linear regression analysis (Pearson correlation coefficient). The concordance of the two methods was analyzed by the Bland-Altman test (18). For each variable the groups were compared by analysis of variance (ANOVA) and by the Wald test, with the level of significance set at $\mathrm{P}<0.05$. Data were analyzed statistically with the Statistical Analysis System software.

\section{Results}

General. It was possible to obtain images adequate for measurement in all animals. 
The mean heart rate after sedation during echocardiography was not significantly different among groups, being $236 \pm 19 \mathrm{bpm}$ for the control group, $236 \pm 21 \mathrm{bpm}$ for the animals with small myocardial infarction, $237 \pm 19 \mathrm{bpm}$ for the animals with mediumsize infarction, and $234 \pm 22 \mathrm{bpm}$ for the animals with large-size infarction.

Myocardial infarction size. By histology, 10 animals $(23.3 \%$ of the total) had small-size myocardial infarction, 10 had medium-size infarction (23.3\% of the total), 13 had large-size infarction $(30.3 \%$ of the total), and 10 (23.3\% of the total) were assigned to the control group. The mean values and standard deviation of myocardial infarct size obtained by histology and by echocardiography for each group are shown in Table 1. Infarct size ranged from 4.8 to $66.6 \%$ when determined by histology and from 5.0 to $69.8 \%$ when determined by echocardiography, with good correlation between the two measurements $(\mathrm{r}=0.88 ; \mathrm{P}<0.05 ; \mathrm{y}$ $=0.9079 x+4.9656$; Figure 1). Bland-Altman analysis showed that the difference between histology and echocardiography was no more than two standard deviation above or below the mean of the two methods, and only two patients were outside this interval (Figure 2).

Table 1. Infarct size determined by echocardiography and histology.

\begin{tabular}{lcccc}
\hline Variable & $\begin{array}{c}\text { Control } \\
(\mathrm{N}=10)\end{array}$ & $\begin{array}{c}\text { Small IS } \\
(\mathrm{N}=10)\end{array}$ & $\begin{array}{c}\text { Medium IS } \\
(\mathrm{N}=10)\end{array}$ & $\begin{array}{c}\text { Large IS } \\
(\mathrm{N}=13)\end{array}$ \\
\hline Histology (\%) & & $17.5 \pm 7.6$ & $35.9 \pm 3.4$ & $46.8 \pm 8.0$ \\
Echocardiography (\%) & & $21.7 \pm 9.7$ & $37.4 \pm 10.6$ & $47.5 \pm 9.6$ \\
LVDd & $0.63 \pm 0.05^{\mathrm{a}}$ & $0.67 \pm 0.07^{\mathrm{a}}$ & $0.78 \pm 0.12^{\mathrm{b}}$ & $0.84 \pm 0.1^{\mathrm{b}}$ \\
LVDa & $0.41 \pm 0.03^{\mathrm{a}}$ & $0.47 \pm 0.07^{\mathrm{a}}$ & $0.56 \pm 0.01^{\mathrm{b}}$ & $0.69 \pm 0.1^{\mathrm{c}}$ \\
FAC & $53.1 \pm 1.5^{\mathrm{a}}$ & $43.7 \pm 9.9^{\mathrm{b}}$ & $34.1 \pm 8.0^{\mathrm{c}}$ & $28.5 \pm 5.6^{\mathrm{c}}$ \\
E wave & $61.3 \pm 4.8^{\mathrm{a}}$ & $56.7 \pm 6.4^{\mathrm{a}}$ & $63.3 \pm 16.1^{\mathrm{a}}$ & $66.9 \pm 13.1^{\mathrm{a}}$ \\
A wave & $31.4 \pm 4.1^{\mathrm{a}}$ & $30.8 \pm 5.5^{\mathrm{a}}$ & $27.9 \pm 8.6^{\mathrm{a}}$ & $16.1 \pm 11.1^{\mathrm{b}}$ \\
E/A ratio & $1.9 \pm 0.1^{\mathrm{a}}$ & $1.9 \pm 0.4^{\mathrm{a}}$ & $2.8 \pm 2.3^{\mathrm{a}}$ & $5.6 \pm 2.7^{\mathrm{b}}$ \\
DT & $42.3 \pm 8.2^{\mathrm{a}}$ & $48.6 \pm 10.2^{\mathrm{a}}$ & $46.3 \pm 11.1^{\mathrm{a}}$ & $41.4 \pm 14.6^{\mathrm{a}}$ \\
IRT & $28.1 \pm 4.3^{\mathrm{a}}$ & $31.7 \pm 3.6^{\mathrm{a}}$ & $33.5 \pm 8.0^{\mathrm{a}}$ & $31.3 \pm 4.2^{\mathrm{a}}$ \\
\hline
\end{tabular}

Data are reported as means \pm SD for each animal group for the following variables: left ventricular diastolic diameter (LVDd, $\mathrm{cm}$ ) and diastolic area $\left(\mathrm{LVDa}, \mathrm{cm}^{2}\right)$, fractional area change (FAC, \%), E and A waves, E/A ratio, deceleration time (DT, ms), and isovolumic relaxation time (IRT, ms). IS = infarct size. Groups followed by the same letter did not differ significantly (ANOVA and Wald test).
Left ventricular diastolic dimensions. The left ventricular diastolic diameters of all groups (means $\pm \mathrm{SD}$ ) are given in Table 1 . There was no statistically significant difference between controls and animals with small-size myocardial infarction or between those with largeand medium-size myocardial infarction $(\mathrm{P}=$ $0.1041)$. The diameter was significantly larger for both large- and medium-size myocardial infarctions compared to control and to animals with small-size myocardial infarction (control vs moderate, $\mathrm{P}=0.0001$; control vs large, $\mathrm{P}=$ 0.0001 ; small vs moderate, $\mathrm{P}=0.0032$; small vs large, $\mathrm{P}=0.0001)$. The diastolic diameter determined by echocardiography correlated significantly with myocardial infarct size both by histology $(\mathrm{r}=0.57 ; \mathrm{P}=0.0005)$ and echocardiography $(\mathrm{r}=0.56 ; \mathrm{P}=0.0001)$. The values of left ventricular diastolic transverse area (mean \pm SD) are given in Table 1. This variable did not differ significantly between controls and animals with small-size myocardial infarction, but was significantly larger in animals with medium-size myocardial infarction than in controls and in animals with small myocardial infarction $(\mathrm{P}=0.0078)$. In animals with large myocardial infarction it was significantly larger than in all other groups (control: $\mathrm{P}=0.0001$; small: $\mathrm{P}=0.0001$; moderate: $\mathrm{P}=$ $0.0020)$. There were significant correlations between end-diastolic area and infarct size by both histology $(\mathrm{r}=0.78 ; \mathrm{P}=0.0001)$ and echocardiography $(\mathrm{r}=0.74 ; \mathrm{P}=0.0001)$.

Left ventricular systolic function. FAC (mean $\pm \mathrm{SD}$ ) was significantly lower in all groups with myocardial infarction compared to control $(\mathrm{P}=0.0045$ for small-size, $\mathrm{P}=$ 0.0001 for medium-size and $\mathrm{P}=0.0001$ for large-size) (Table 1). FAC did not differ significantly between the medium and large myocardial infarction groups, but the values for both groups were significantly lower than those for the group with small-size myocardial infarction (small vs moderate, $\mathrm{P}<0.004$; small vs large, $\mathrm{P}<0.0001)$. FAC correlated well with myocardial infarct size both by echocardiography $(r=-0.87 ; \mathrm{P}<0.00001)$ and histol- 
ogy $(\mathrm{r}=-0.78 ; \mathrm{P}<00001 ;$ Figure 3$)$.

Left ventricular diastolic function. The mean values of $\mathrm{E}$ and $\mathrm{A}$ wave velocities, $\mathrm{E} /$ A ratio, deceleration time and isovolumic deceleration time for all groups of animals are given in Table 1. There was no difference in $\mathrm{E}$ wave velocity between groups. The A wave was significantly smaller in animals with large myocardial infarction as compared to the others (control: $\mathrm{P}=0.0001$; small: $\mathrm{P}=0.0001$; medium: $\mathrm{P}=0.0018)$, but no difference was observed between the medium-size and small-size myocardial infarction groups and the control group. The E/A ratio (Figures 4 and 5) of animals with large myocardial infarction was significantly higher than observed in all other groups (control: $\mathrm{P}=0.0001$; small: $\mathrm{P}=0.0001$; medium: $\mathrm{P}=0.0014$ ), but no statistical difference was observed between the control

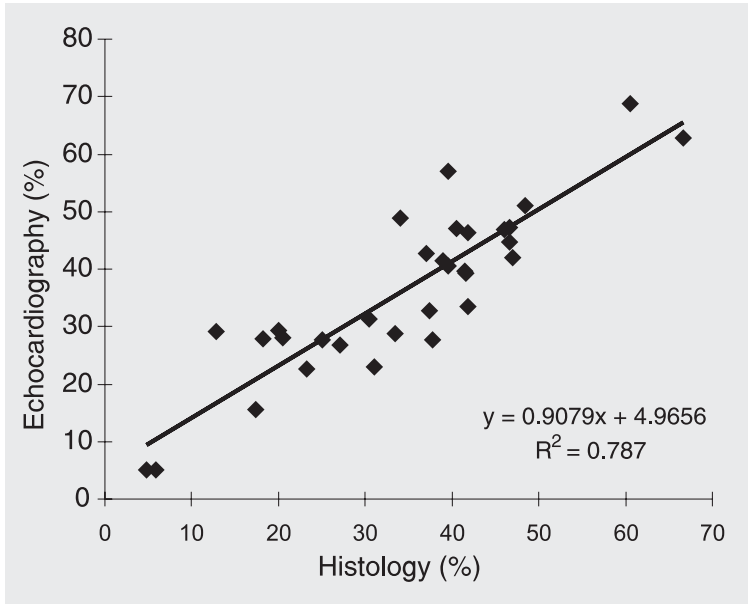

Figure 1. The correlation between infarct size determined by histology and echocardiography.

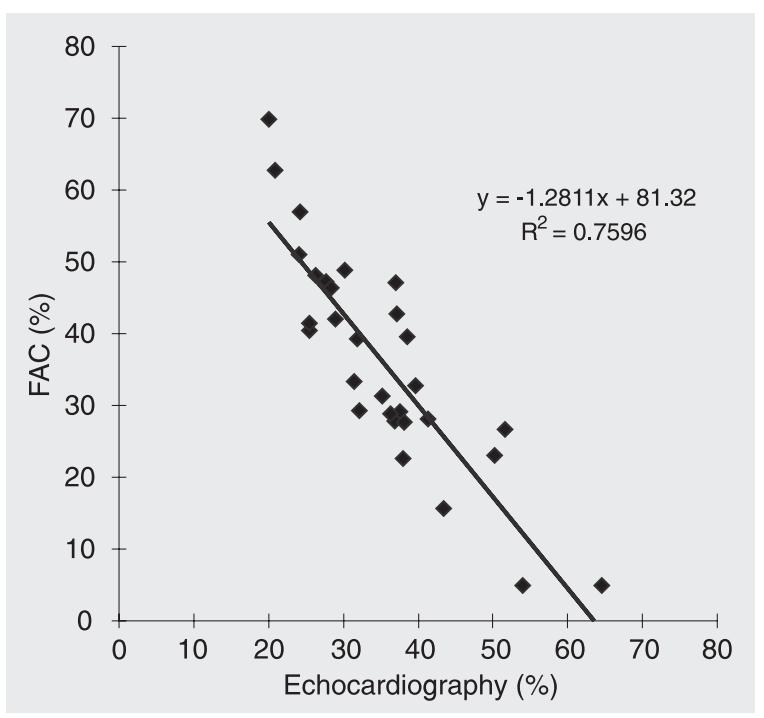

Figure 3. The correlation between fractional area change (FAC) and infarct size determined by echocardiography.

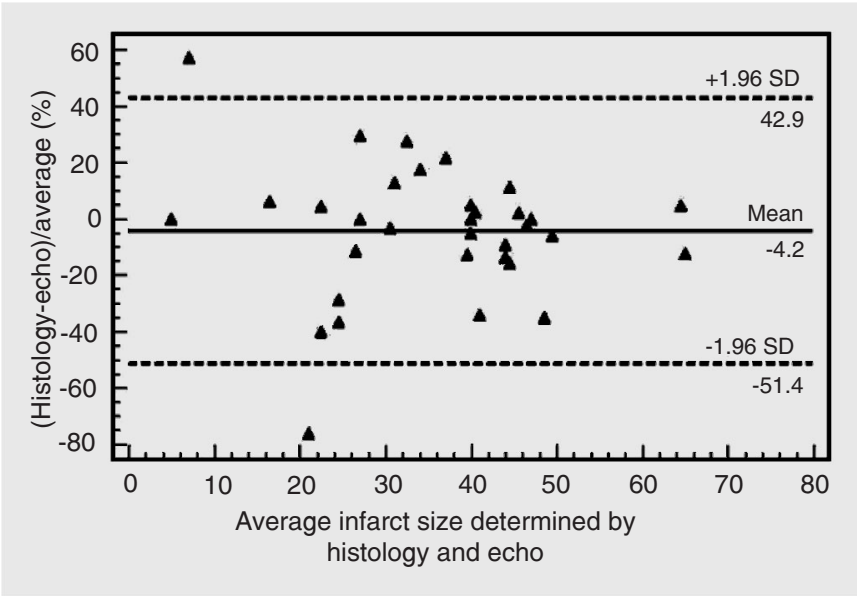

Figure 2. Bland and Altman plot comparing the measurements of infarct size by histology and echocardiography (echo). SD = standard deviation.

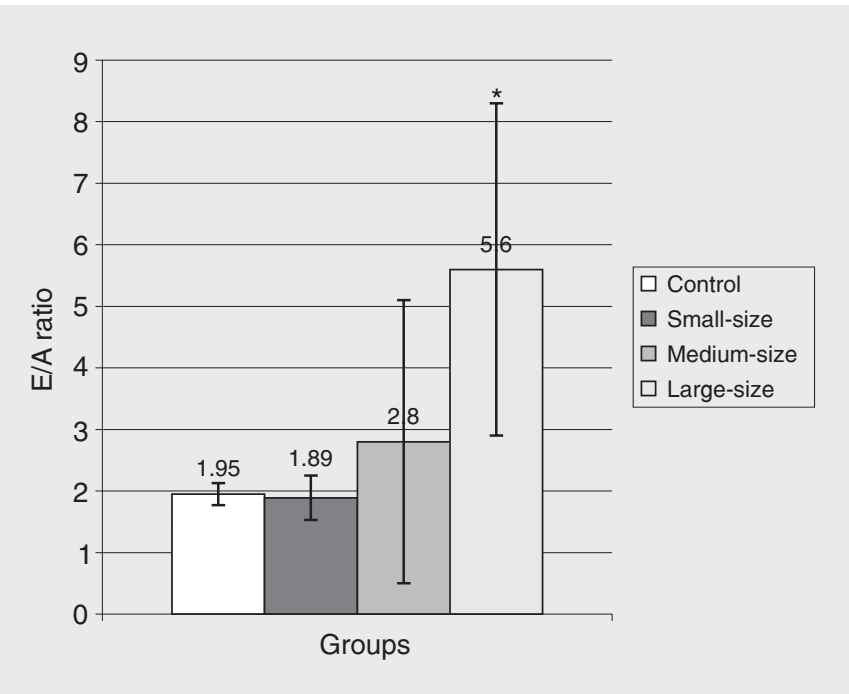

Figure 4. Bar graph showing the mean E/A ratios of all groups. *A significant difference was observed only for large-size myocardial infarct compared to other groups (ANOVA and Wald test). 
and small- and medium-size myocardial infarction groups. Also, there was no statistically significant difference between groups for isovolumetric relaxation time or deceleration time (Table 1).

\section{Discussion}

Baily et al. (12) first reported the use of echocardiography to analyze the rat heart with myocardial infarction. To evaluate the infarct size, these investigators used a score system by which a point was assigned to each akinetic or dyskinetic segment among the four segments of the left ventricle associated or not with chamber dilation. When they compared this score system to histological measurements of infarct size the authors concluded that echocardiography could be used to estimate myocardial infarct size. Litwin et al. (15) studied rats with large myocardial infarction and estimated the infarct size by measuring the percentage of left ventricular endocardial circumference that was akinetic on a freeze-frame image, apparently on only one transverse plane. They found no difference in the size of myocardial infarction estimated by this method and histology. In a study by another group, only rats with moderate-size myocardial infarction were included. These investigators used an echocardiographic method similar to that

Figure 5. a, Velocity flow profile of a control rat with normal $E$ and $A$ wave velocities and $E / A$ ratio. $b$, Velocity flow profile of a rat with a large myocardial infarction with high $E$ and low $A$ wave velocities and a high E/A ratio that may represent a restrictive pattern. a

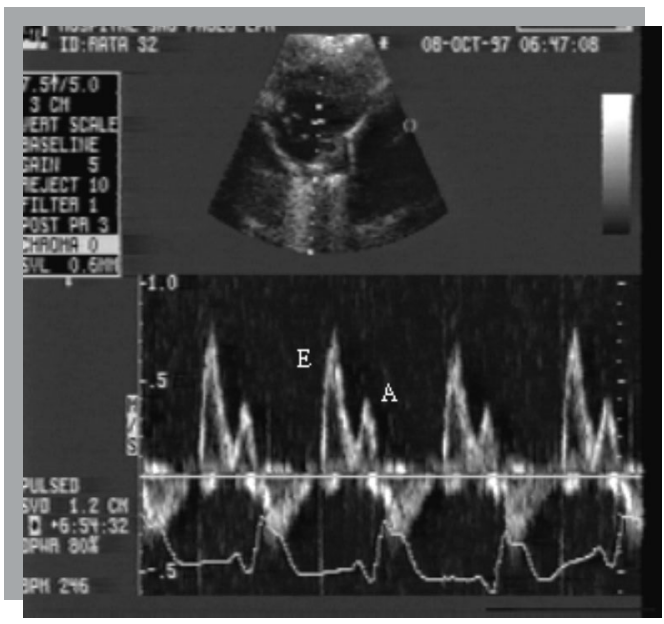

used in the present study, but the measurements were performed only in the middle cavity, and no differences were found compared to histology (13).

In the present study, the histological measurement of myocardial infarct size on several transverse planes was used for echocardiography $(1,2)$. The mean measurements of the myocardial scar at the endocardial and epicardial borders were used, as also done by other investigators, who consider the method to be a precise estimate of the total myocardial infarct size. Measurements on three transverse planes were performed by echocardiography at the endocardial border only, and were found to be significantly correlated $(\mathrm{r}=0.88 ; \mathrm{P}<0.05)$ with the measurements obtained by histology, with a difference not exceeding two standard deviations for most animals. However, echocardiography slightly overestimated the myocardial infarct size compared to histology. This may have occurred because both akinesis and hypokinesis were considered to indicate a possible area of myocardial infarction by echocardiography, while only areas with fibrosis, possibly akinetic, were considered to indicate infarction by histology. These regions of hypokinesis around the area of myocardial infarction fibrosis may have increased the myocardial infarction size by echocardiography. This technique is very b

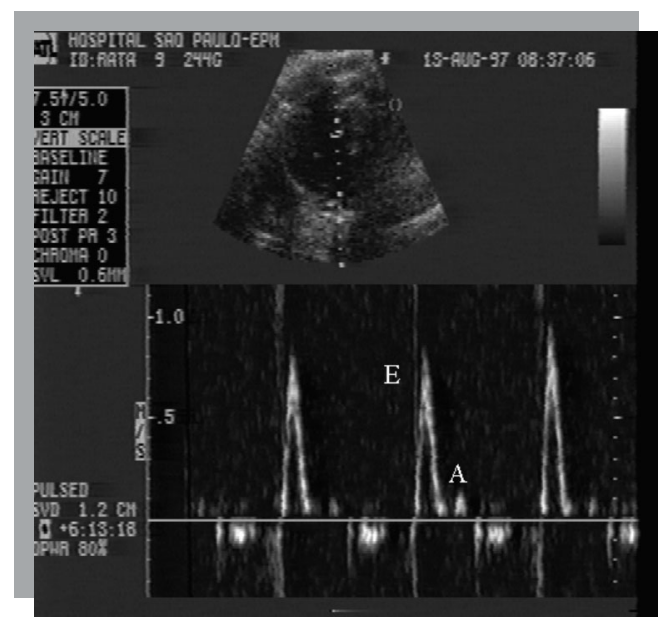


sensitive for detecting segmental wall motion abnormalities in humans. In the present study, all myocardial infarctions detected by echocardiography were confirmed by histology, and none of the sham-operated animals (control) showed infarction by echocardiography or histology. These findings indicate that the echocardiographic method used in the present study may be a good alternative for the identification and quantification of infarct size in rats, with the advantage of being applicable to living animals, an important factor for follow-up or intervention studies. Recently, a study on mice using the same process as that employed in the present study also obtained a high correlation coefficient between histology and echocardiography (19).

Left ventricular size was evaluated by the diastolic anteroposterior diameter and diastolic transverse area. The diastolic diameter was significantly larger in animals with medium and large myocardial infarction compared to control and to animals with small-size myocardial infarction, and showed a modest but significant correlation with infarct size by echocardiography and histology. The mean diastolic area measured on three transverse planes by echocardiography did not differ significantly between animals with small myocardial infarction and controls, but considerably significant differences were observed among the other groups. The correlation coefficients with infarct size determined by echocardiography and histology ( 0.78 and 0.74 , respectively) were better than for the diastolic diameter $(0.57$ and 0.56 , respectively). These superior results may be due to the fact that the diastolic area, the result of the mean of three transverse planes, may represent the volume of the chamber more than the diameter, which was measured on only one plane. In dilated ventricles with segmental wall motion abnormalities and irregular geometry, one diameter may not represent the actual size of the ventricle, while the diastolic area on three planes may be closer to the diastolic volume than the diameter of the chamber.

The left ventricular systolic function of the animals was estimated by the FAC, which was also the mean value for three transverse planes. The value was significantly smaller in animals with myocardial infarction than in controls, but did not differ significantly between animals with large- and moderatesize myocardial infarction. The FAC also showed a good and significant negative correlation with infarct size by echocardiography $(\mathrm{r}=-0.87)$ and histology $(\mathrm{r}=-0.78)$, as also observed in other studies $(13,14)$. Due to irregular chamber geometry and segmental wall motion abnormalities, as pointed out about the left ventricular size, the systolic function was not estimated by the fractional shortening of the anteroposterior diameters. It would be interesting to use the Simpson method to estimate left ventricular systolic function, but, since in the four-chamber view the echocardiographic system used (7.5-MHz transducer) did not allow good definition of the endocardial borders in most animals, the method was not used in the present study. It is possible that with the use of more recent and advanced echocardiographic systems, with 12-MHz transducers, better image quality may be obtained from all planes.

In the present study, diastolic function was analyzed by the mitral diastolic flow velocity curve based on $\mathrm{E}$ and $\mathrm{A}$ waves, $\mathrm{E} / \mathrm{A}$ ratio and deceleration time, as well as the isovolumic relaxation time. There was a significant increase in the E/A ratio and a significant decrease in A wave only in the group of animals with large myocardial infarction. Although no significant change of deceleration time was found, it is possible that the association of a significantly higher E/A ratio in the animals with large myocardial infarction may represent a trend toward restrictive physiology with increased left ventricular diastolic pressure (20). The high E wave velocity in the animals with large myocardial infarction is due to an increased 
pressure gradient between left atrium and left ventricle at early diastole, while the decrease of A wave velocity may be associated with increased left ventricular end-diastolic pressure with a reduced pressure gradient between the left atrium and left ventricle. None of the animals in the present study showed a profile of mitral diastolic flow velocity indicating impaired relaxation and, since the pulmonary vein flow velocity or mitral annular velocity profiles were not recorded, it was not possible to identify a pseudonormal mitral flow velocity pattern. The deceleration time and the isovolumic relaxation time did not change significantly among the various animal groups. The high heart rate of the animals and the short duration of these time intervals may be the reasons for the lack of detection of larger variations.

The heart rate of the rats after anesthesia for echocardiography was lower than expected for awake animals, but no difference was observed among groups. Although some physiologic implications may occur with relative bradycardia due to anesthesia with ketamine and xylazine, echocardiography has some advantages. An elevated heart rate may compromise the image quality, accu- racy and reproducibility of measurements, particularly those obtained by the Doppler technique. Parameters of diastolic function, particularly $\mathrm{E}$ and $\mathrm{A}$ waves and $\mathrm{E} / \mathrm{A}$ ratio, may be affected by heart rate, but Doppler echocardiography was performed in all groups of animals under the same physiologic conditions, and possibly did not influence the results.

In the present study, the technical principles used to produce myocardial infarction in rats were similar to those used by others $(1,2,13,14)$. However, while most of the other studies were conducted on male rats, the present study used only female Wistar rats. Although some anatomical and physiological cardiac differences may eventually exist between genders and rat strains, which would limit the comparison with other studies, since all animals in the present study had similar characteristics this may not have influenced our results.

On the basis of the present data, we conclude that Doppler echocardiography is a valid technique to estimate myocardial infarct size and to evaluate the left ventricular morphology and function of rats with myocardial infarction of different sizes.

\section{References}

1. Pfeffer AM, Pfeffer JM, Fishbein MC et al. (1979). Myocardial infarct size and ventricular function in rats. Circulation Research, 44: 503512.

2. Spadaro J, Fishbein MC, Hare C et al. (1980). Characterization of myocardial infarction in the rat. Archives of Pathology and Laboratory Medicine, 104: 179-183.

3. Fletcher PJ, Pfeffer JM, Pfeffer MA et al. (1981). Left ventricular diastolic pressure-volume relations in rats with healed myocardial infarction: effects on systolic function. Circulation Research, 49: 618-626.

4. Pfeffer JM, Pfeffer MA, Fletcher PJ et al. (1991). Progressive ventricular remodeling in rat with myocardial infarction. American Journal of Physiology, 260: H1406-H1414.

5. Fishbein MC, MacLean D \& Maroko PR (1978). Experimental myocardial infarction in the rat: qualitative and quantitative changes during pathologic evolution. American Journal of Pathology, 90: 5770

6. Litwin SE, Litwin CM, Raia TE et al. (1991). Contractility and stiff- ness of noninfarcted myocardium after coronary ligation in rats: effects of chronic angiotensin converting enzyme inhibition. Circulation, 83: 1028-1037.

7. Litwin SE, Katz SE, Morgan JP et al. (1996). Long-term captopril treatment improves diastolic filling more than systolic performance in rats with large myocardial infarction. Journal of the American College of Cardiology, 28: 773-781.

8. Kanashiro RM, Nozawa E, Murad N et al. (2002). Myocardial infarction scar plication in the rat: cardiac mechanics in an animal model for surgical procedures. Annals of Thoracic Surgery, 73: 1507-1513.

9. Kondo I, Ohmori K, Oshita A et al. (2004). Treatment of acute myocardial infarction by hepatocyte growth factor gene transfer. The first demonstration of myocardial transfer of a "functional" gene using ultrasonic microbubble destruction. Journal of the American College of Cardiology, 44: 644-653.

10. Scorsin M, Hagege AA, Marotte F et al. (1997). Does transplantation of cardiomyocytes improve function of infarcted myocardium? Circulation, 96 (Suppl): II-188-II-193. 
11. Cheitlin MD, Alpert JS, Armstrong WF et al. (2003). ACC/AHA guidelines for the clinical application of echocardiography: executive summary. A report of the American College of Cardiology/ American Heart Association Task Force on Practice Guidelines (Committee on Clinical Application of Echocardiography). Developed in collaboration with the American Society of Echocardiography. Journal of the American College of Cardiology, 29: 862-879.

12. Baily RG, Lehman CG, Gubin SS et al. (1993). Non-invasive assessment of ventricular damage in rats with myocardial infarction. Cardiovascular Research, 27: 851-853.

13. Burrel LM, Chan R, Philips AP et al. (1996). Validation of an echocardiographic assessment of cardiac function following moderate size myocardial infarction in the rat. Clinical and Experimental Pharmacology and Physiology, 23: 570-572.

14. Hagar MJ, Matthew R \& Kloner AR (1995). Quantitative two-dimensional echocardiographic assessment of regional wall motion during transient ischemia and reperfusion in the rat. Journal of the American Society of Echocardiography, 8: 162-174.

15. Litwin SE, Katz SE, Morgan PG et al. (1994). Serial echocardiographic assessment of left ventricular geometry and function after large myocardial infarction in the rat. Circulation, 89: 345-354.

16. Sahn DJ, DeMaria A, Kisslo J et al. (1978). Recommendations regarding quantitation in M-mode echocardiography: survey of echocardiographic measurements. Circulation, 58: 1072-1083.

17. Schiller NB, Shah PM, Crawford M et al. (1989). Recommendations for quantitation of the left ventricle by two-dimensional echocardiography. Journal of the American Society of Echocardiography, 2: 358-367.

18. Bland JM \& Altman DG (1978). Statistical methods for assessing agreement between two methods of clinical measurements. Lancet, 8: 307-310.

19. Kanno S, Lerner DL, Schuessler RB et al. (2002). Echocardiographic evaluation of ventricular remodeling in a mouse model of myocardial infarction. Journal of the American Society of Echocardiography, 15: 601-609.

20. Oh JK, Ding ZP, Gersh BJ et al. (1992). Restrictive left ventricular diastolic filling identifies patients with heart failure after acute myocardial infarction. Journal of the American Society of Echocardiography, 5: 497-503. 